\title{
The electrostatic potential profile along a biased molecular wire: A model quantum- mechanical calculation
}

Stéphane Pleutin, Hermann Grabert, Gert-Ludwig Ingold, and Abraham Nitzan

Citation: The Journal of Chemical Physics 118, 3756 (2003); doi: 10.1063/1.1539863

View online: https://doi.org/10.1063/1.1539863

View Table of Contents: http://aip.scitation.org/toc/jcp/118/8

Published by the American Institute of Physics

\section{Articles you may be interested in}

On the electrostatic potential profile in biased molecular wires

The Journal of Chemical Physics 117, 10837 (2002); 10.1063/1.1522406

Molecular wire conductance: Electrostatic potential spatial profile

The Journal of Chemical Physics 112, 6834 (2000); 10.1063/1.481258

Generalized Formula for the Electric Tunnel Effect between Similar Electrodes Separated by a Thin Insulating Film

Journal of Applied Physics 34, 1793 (1963); 10.1063/1.1702682

Electron conduction in molecular wires. I. A scattering formalism

The Journal of Chemical Physics 101, 6849 (1994); 10.1063/1.468314

Quantum transport with two interacting conduction channels

The Journal of Chemical Physics 138, 174111 (2013); 10.1063/1.4802587

Interatomic inelastic current

The Journal of Chemical Physics 146, 092322 (2017); 10.1063/1.4975320

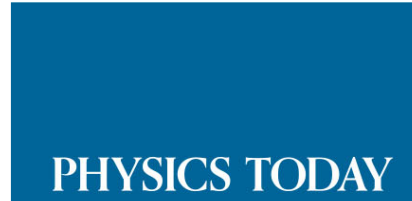




\title{
The electrostatic potential profile along a biased molecular wire: A model quantum-mechanical calculation
}

\author{
Stéphane Pleutin and Hermann Grabert \\ Physikalisches Institut, Albert-Ludwigs-Universität, Hermann-Herder-Straße 3, D-79104 Freiburg, Germany \\ Gert-Ludwig Ingold \\ Institut für Physik, Universität Augsburg, Universitätsstraße 1, D-86135 Augsburg, Germany
}

Abraham Nitzan

School of Chemistry, The Sackler Faculty of Science, Tel Aviv University, 69978 Tel Aviv, Israel

(Received 4 October 2002; accepted 27 November 2002)

\begin{abstract}
We study the electrostatic potential of a molecular wire bridging two metallic electrodes in the limit of weak contacts. With the use of a tight-binding model including a fully three-dimensional treatment of the electrostatics of the molecular junction, the potential is shown to be poorly screened, dropping mostly along the entire molecule. In addition, we observe pronounced Friedel oscillations that can be related to the breaking of electron-hole symmetry. Our results are in semiquantitative agreement with recent state-of-the-art $a b$ initio calculations and point to the need of a three-dimensional treatment to properly capture the behavior of the electrostatic potential. Based on these results, current-voltage curves are calculated within the Landauer formalism. It is shown that Coulomb interaction partially compensates the localization of the charges induced by the electric field and consequently tends to suppress zones of negative differential resistance. (C) 2003 American Institute of Physics. [DOI: 10.1063/1.1539863]
\end{abstract}

\section{INTRODUCTION}

Due to important technical progress, the field of molecular electronics, born in the mid-70s with the proposal of Aviram and Ratner to use single organic molecules as rectifiers, ${ }^{1}$ receives rapidly growing interest. ${ }^{2-4}$ Indeed, new fabrication methods and probes now enable individual molecules or small numbers of them to be connected to macroscopic electrodes. ${ }^{2,5-8}$ Among these methods, one may cite, for instance, the break-junction technique ${ }^{5,6,8}$ and the use of a conducting atomic-force microscope (AFM) to contact molecules absorbed on a metallic surface. ${ }^{7}$

On the theoretical side, the problem posed by these experimental works is highly challenging. We are facing a nonequilibrium many-body problem where, moreover, the coupling to a phonon bath may also be of importance. Up to now, most of the studies have focused on the coherent regime and the Landauer approach has been employed to obtain the conductance from $a b$ initio or semiempirical models. ${ }^{3}$ Important inelastic processes were included only within simple models ${ }^{9-13}$ and much further progress is needed before one may hope to reach a satisfactory understanding of the problem.

The exact number of molecules contacted by the leads remains for a large part uncontrolled in the experimental setups cited above. ${ }^{2,8}$ In theoretical modeling it is convenient to assume that a single organic molecule bridges two semiinfinite metallic electrodes (cf. Fig. 1). Another important experimental aspect is the fact that current-voltage characteristics are measured with applied voltages up to a few volts, values which bring us well away from the linear regime.

In this context, a central question concerns the electro- static potential profile of a biased molecular wire. The importance of this issue was first demonstrated by Datta and co-workers. ${ }^{14,15}$ Using semiempirical models, they have shown that different choices of the electrostatic profile have a profound effect on the current-voltage characteristics of a molecular junction. For instance, the transport properties are strongly modified depending on whether the potential drop occurs at the interface between molecule and electrode or along the molecular wire. In fact, it is natural to assume that even the details of the potential shape have a considerable effect on molecular conductance.

Recently, a few works along that line have been reported. ${ }^{16-18}$ They give us a rather ambiguous view of this fundamental problem. Model calculations involving selfconsistent solutions of the coupled Poisson and Schrödinger equations suggest that the potential drop occurs mainly at the interface between the molecule and the electrodes. ${ }^{16}$ Within the molecule, the electrostatic potential is then found to be essentially flat. Screening appears to be very efficient within this approach, and the final conclusions are in agreement with some previous investigations. ${ }^{14,15}$ However, these model calculations involve a drastic approximation: instead of solving the full Poisson equation, the authors of Ref. 16 have used a one-dimensional version of it. Implicitly, it is then assumed that the lateral dimensions of the molecule are much larger than the screening length. For quasi-onedimensional systems with lateral dimensions of the order of a few angstroms, such as the organic molecules used in recent experimental work, this approximation is clearly questionable. ${ }^{19}$ Indeed, recent state-of-the-art $a b$ initio calculations on carbon and gold chains show a quite different picture. ${ }^{17,18}$ In these works, the potential drop occurs not 
only at the interface but rather along the entire molecule. Moreover, the local potential is found to display pronounced Friedel oscillations. Contrary to previous results, screening appears to be rather inefficient, even for metallic wires.

We are then left with two different pictures, and it becomes clear that a full understanding of the electrostatic potential profile in biased molecular wires or metallic constrictions is still lacking. In this work, we readdress the problem following the approach by Mujica et al. ${ }^{16}$ We perform model calculations and solve the coupled set of two equations: the Poisson equation for the electrostatics and the Schrödinger equation for the electronic structure. In this respect, our work is similar to that of Mujica et al. ${ }^{16}$ However, the calculation is modified in ways that we believe to be essential. In particular, we treat the real three-dimensional Poisson equation. As recently discussed by us, ${ }^{19}$ we expect that this proper handling of the electrostatic problem changes the qualitative behavior: The potential is poorly screened and falls off substantially along the molecule. Indeed, the electrostatic potential profile of the model calculations presented here is in semiquantitative agreement with the $a b$ initio results reported in Refs. 17 and 18. However, while $a b$ initio calculations are involved and intricate enough to leave the underlying physics essentially obscure, our model includes only the ingredients necessary to capture the correct screening effects, and consequently our calculations are rather economic in time. We thus believe that our approach may help to gain further insight into the difficult problem of understanding transport through molecules, i.e., at a scale where quantum effects are prominent. A forthcoming work by Ghosh et al. ${ }^{20}$ reaches conclusions similar to those presented here.

In Sec. II, our model Hamiltonian is introduced. The electronic density in the absence of a bias potential is then studied in Sec. III, using exact diagonalization and HartreeFock calculations that are shown to agree reasonably well with each other. The electrostatic profile is then calculated in Sec. IV at the Hartree-Fock level. Finally, the current-voltage characteristics are discussed in Sec. V.

\section{MODEL HAMILTONIAN}

\section{A. Coulomb interaction including image charges}

The physical problem posed by a molecular wire between two infinite metallic reservoirs is far too complicated to be solved exactly and, to proceed, several approximations are necessary. First, we assume that the surfaces of the two electrodes are infinite planes (cf. Fig. 1). Second, the molecule is assumed to be weakly connected to the metallic electrodes so that their chemical constitution is unimportant. This is certainly not fulfilled for some of the wires examined experimentally, with covalent bonds between molecule and electrode. Finally, we assume the characteristic time scale for electronic processes in the electrodes to be much shorter than the transit time of electrons in the wire. The electrodes can then be treated as equipotential surfaces, and the Schrödinger equation is solved under these potential boundary conditions. We are then within the same framework used in Ref. 16, but proceed differently. We first determine the Coulomb interac-

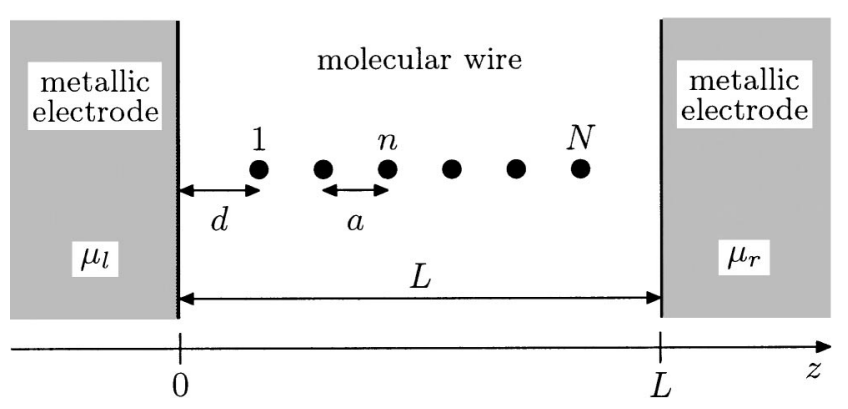

FIG. 1. Idealized molecular junction. A molecular wire, modeled by a finite one-dimensional lattice, bridges two metallic electrodes with surfaces assumed to be infinite planes. The tunneling contacts, effective only at the two molecular end sites indicated by 1 and $N$, are assumed to be weak. The chemical potentials in the left and right electrode are denoted by $\mu_{l}$ and $\mu_{r}$, respectively.

tion potential which includes the image charges due to the metallic leads, keeping its three-dimensional character. Then, we solve the electronic wire problem.

The Coulomb interaction energy reads

$$
W=\frac{1}{2} \int d^{3} \mathbf{r} \int d^{3} \mathbf{r}^{\prime} \rho(\mathbf{r}) \rho\left(\mathbf{r}^{\prime}\right) \varphi\left(\mathbf{r}, \mathbf{r}^{\prime}\right),
$$

where $\rho(\mathbf{r})$ is the charge density in the wire and $\varphi\left(\mathbf{r}, \mathbf{r}^{\prime}\right)$ is the potential at point $\mathbf{r}=(x, y, z)$ produced by the charge located at point $\mathbf{r}^{\prime}=\left(x^{\prime}, y^{\prime}, z^{\prime}\right)$. The Coulomb potential is the solution of the Poisson equation

$$
\Delta_{\mathbf{r}} \varphi\left(\mathbf{r}, \mathbf{r}^{\prime}\right)=-4 \pi U \delta\left(\mathbf{r}-\mathbf{r}^{\prime}\right),
$$

where for convenience we have introduced a factor $U$ measuring the strength of the electron-electron interaction. In the absence of the metallic electrodes the solution of (2) is given by the standard Coulomb potential

$$
\varphi_{0}\left(\mathbf{r}, \mathbf{r}^{\prime}\right)=\frac{U}{\left|\mathbf{r}-\mathbf{r}^{\prime}\right|} .
$$

In the setup depicted in Fig. 1, we require the potential in the absence of an external bias to vanish on the surfaces of the metallic electrodes, i.e., at $z=0$ and $z=L$. A solution of the Poisson equation with these particular boundary conditions is found using the standard method of image charges

$$
\varphi\left(\mathbf{r}, \mathbf{r}^{\prime}\right)=\sum_{p=-\infty}^{+\infty}\left[\varphi_{0}\left(\mathbf{r}+2 p L \hat{\mathbf{z}}, \mathbf{r}^{\prime}\right)-\varphi_{0}\left(2 p L \hat{\mathbf{z}}-\mathbf{r}, \mathbf{r}^{\prime}\right)\right]
$$

where $L$ is the distance between the two electrodes (cf. Fig. 1) and $\hat{\mathbf{z}}$ the unit vector along the molecular axis.

$\varphi\left(\mathbf{r}, \mathbf{r}^{\prime}\right)$ is a genuine three-dimensional Coulomb potential including the effects of the two semi-infinite metallic electrodes. The electrostatics of the molecular junction is then governed by this potential. It remains to construct and solve the Schrödinger equation for the molecular wire.

\section{B. Tight-binding model including image charges}

In the following, we will mainly be concerned with conjugated molecules and, in particular, with their low-energy properties. An appropriate description can then be given by 
an effective tight-binding Hamiltonian for the $\pi$ electrons only. ${ }^{21,22}$ In addition, since there exist $a b$ initio results for short chains of gold atoms, ${ }^{18}$ it is also of interest to study systems with electrons in $s$ orbitals.

We therefore attach Gaussian-type orbitals of the form

$$
\phi_{n}(\mathbf{r})=A_{s} x^{s} \exp \left[-\alpha\left[x^{2}+y^{2}+\left(z-z_{n}\right)^{2}\right]\right],
$$

to each atomic site $n$. This allows us to model both $s$ orbitals with $s=0$ and $p$ orbitals with $s=1$. The center of the orbital, $z_{n}=d+(n-1) a$, depends on the distance $d$ between each electrode and the molecule as well as the lattice constant $a$ in the molecule (cf. Fig. 1). The parameter $\alpha$ determines the spread of the state and gives an estimate of the electronic density. Finally, the normalization constants for $s$ - and $p$ orbitals are given by $A_{0}=(2 \alpha / \pi)^{3 / 4}$ and $A_{1}=2(2 / \pi)^{3 / 4} \alpha^{5 / 4}$, respectively.

The explicit form (5) of the orbitals allows us to determine the effective parameters entering the tight-binding model. In the following, we adopt the "zero differential overlap" (ZDO) approximation ${ }^{21-23}$

$$
\phi_{n}^{*}(\mathbf{r}) \phi_{m}(\mathbf{r})=\left|\phi_{n}(\mathbf{r})\right|^{2} \delta_{n, m},
$$

which remains valid as long as the orbitals $\phi_{n}$ are strongly localized on the atomic sites $n$. It implies orthogonality between the Gaussian orbitals on different sites and, most importantly, leads to a drastic reduction of nonvanishing matrix elements of the Coulomb operator since only the two center integrals are retained in the final model. In particular, no exchange integrals will appear. These integrals, involving a differential overlap, are usually negligibly small compared to the Coulomb integrals. Moreover, we approximate the positive cores by point charges localized at the atomic sites $m$ with coordinates $\left(0,0, z_{m}\right)$. Then, the energy of an electron localized at site $n$ due to all positive core charges and their images becomes

$$
\epsilon_{n}=-U \sum_{m=1}^{N} \int d^{3} \mathbf{r}\left|\phi_{n}(\mathbf{r})\right|^{2} \varphi\left(\mathbf{r}, z_{m} \hat{\mathbf{z}}\right) .
$$

Within the ZDO approximation the only finite Coulomb matrix elements are related to the interaction energy between electrons localized at sites $n$ and $n^{\prime}$

$$
U_{n, n^{\prime}}=U \int d^{3} \mathbf{r} \int d^{3} \mathbf{r}^{\prime}\left|\phi_{n}(\mathbf{r})\right|^{2} \varphi\left(\mathbf{r}, \mathbf{r}^{\prime}\right)\left|\phi_{n^{\prime}}\left(\mathbf{r}^{\prime}\right)\right|^{2} .
$$

It is worthwhile to notice that the interaction terms (7) and (8) depend on the position $n$ along the chain due to the image charges but also because of the finite size of the molecular wire.

Within the ZDO approximation kinetic energy contributions vanish as a consequence of (6). To lowest order, the overlap leads to a constant shift of the on-site energy $\epsilon_{n}$ that may be disregarded and to nearest-neighbor hopping. The hopping matrix element $t$ cannot be evaluated directly within the ZDO and, therefore, has to be treated as a parameter of the model. ${ }^{21-23}$ However, it is possible to relax the approximation $^{23}$ and estimate the hopping matrix elements from (5). Doing so, we have found that their dependences on $n$ are not pronounced, and, moreover, this kind of more sophisticated treatment would not change qualitatively our final conclusions. Therefore, we assume the hopping matrix elements, $t$, to be constant along the chain, and use the ratio $U / t$ as a parameter to examine the importance of electronelectron interaction.

With the parameters just discussed, we obtain a description of the electrons in terms of an effective tight-binding model which includes long-range Coulomb interaction ${ }^{21,22}$

$$
\begin{aligned}
H= & \sum_{n, \sigma}\left(\epsilon_{n}+v_{n}\right) c_{n, \sigma^{\prime}}^{\dagger} c_{n, \sigma}+\sum_{n, \sigma} t\left(c_{n+1, \sigma}^{\dagger} c_{n, \sigma}+\text { h.c. }\right) \\
& +\frac{1}{2} \sum_{n, n^{\prime}, \sigma, \sigma^{\prime}} U_{n, n^{\prime}} c_{n, \sigma^{\dagger}}^{\dagger} c_{n^{\prime}, \sigma^{\prime}}^{\dagger} c_{n^{\prime}, \sigma^{\prime}} c_{n, \sigma} .
\end{aligned}
$$

$c_{n, \sigma}^{\dagger}\left(c_{n, \sigma}\right)$ are the usual creation (annihilation) operators for an electron with spin $\sigma$ in the local state $\phi_{n}$. In the first term, we have accounted for an additional shift of the local potential due to an external bias. With the chemical potentials $\mu_{l}$ and $\mu_{r}$ in the left and right electrode, respectively, the shift at site $n$ is given by

$$
v_{n}=\mu_{l}+\frac{\mu_{r}-\mu_{l}}{L} z_{n} .
$$

The resulting tight-binding model (9) is mostly defined by the geometry of the molecular junction. The only other parameters are the energies $t$ and $U$, which determine the strength of the kinetic and Coulomb energies, respectively. It is usually believed that conjugated molecules lie in an intermediate regime where $U / t=1, \ldots, 4 .^{21,22}$

\section{ELECTRON DENSITY WITHOUT BIAS POTENTIAL}

Consider first the situation without bias potential, $\mu_{l}$ $=\mu_{r}$ or $v_{n}=0$. We analyze for this case the electron density at equilibrium in the absence of electron transfer between the molecule and the leads. In all the calculations presented here, we assume the chains to be electrically neutral with on average one electron per site. Because of the electron spin, this corresponds to the half-filled case. Furthermore, we restrict ourselves to the ground state of the system.

We have done exact diagonalization studies for chains of up to 12 sites. Results for the charge density of a chain with 12 sites shown in Fig. 2 are typical for other cases studied. Two main features can easily be seen. (i) The electron density is nonuniform: the electrons have a tendency to shift towards the middle of the chain. (ii) Because of this nonuniformity, substantial Friedel oscillations occur across the wire. These features can be explained by invoking electron-hole symmetry as shown below.

For the case of a half-filled band it has long been known that the electron density is uniform, i.e., does not depend on the site index $n$, for models defined on bipartite lattices in such a way that electron-hole symmetry is fulfilled. This theorem was first discovered for the ground state of the freeelectron (Hückel) model $^{24}$ and later extended to some interacting systems. ${ }^{25}$ More recently, a generalization to canonical and grand canonical ensembles was proven for a large class of models. ${ }^{26}$ Mathematically, the theorem applies to models invariant with respect to the transformation $c_{n, \sigma}^{\dagger}$ $\rightarrow(-1)^{n} c_{n, \sigma}$. 


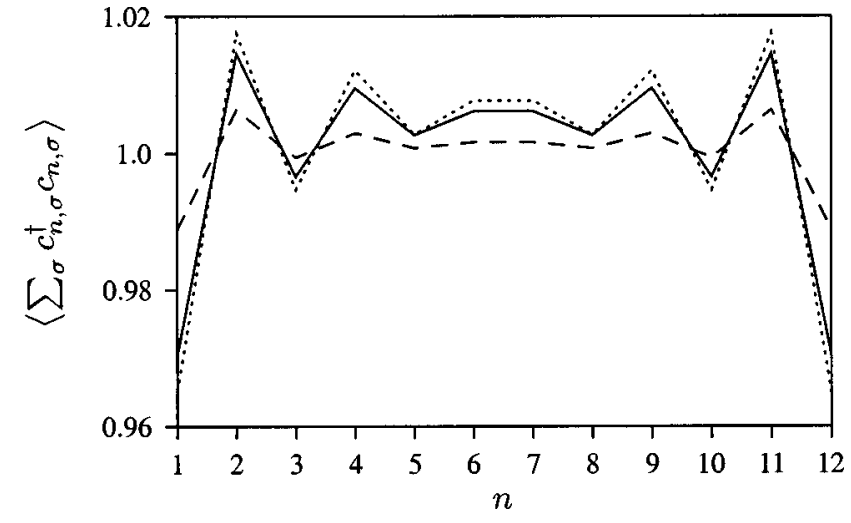

FIG. 2. Electronic density without bias potential for a molecular wire of carbon $(s=1)$ with $N=12$ sites. The parameters are $\alpha=4.5 / a^{2}$ and $U=t$. The full curve is the exact result for a molecule-electrode distance of $d$ $=2 a$. The dotted curve is for the same geometry but at Hartree-Fock level. The dashed curve is the exact result for the case without metallic electrodes, $d \rightarrow \infty$.

Applying this transformation, we find that the Hamiltonian of the molecular wire (9) is invariant only if the equality

$$
\epsilon_{n}=-\frac{1}{2} \sum_{m=1}^{N} U_{n, m}\left(1-\frac{\delta_{n, m}}{2}\right)+K
$$

is fulfilled, where $K$ is a constant. This implies that the electron-ion interaction is essentially compensated, up to a constant term, by the repulsive electron-electron interaction. Notice that allowing for hopping matrix elements that depend on the position in the chain would not change this equality. Indeed, a term like $\sum_{n, \sigma} t_{n, n+1}\left(c_{n+1, \sigma}^{\dagger} c_{n, \sigma}\right.$ $+c_{n, \sigma}^{\dagger} c_{n+1, \sigma}$ ) remains unchanged when the electron-hole transformation is applied. From this point of view, it is not necessary to go beyond the ZDO approximation.

The equality (11) can be satisfied only in very particular cases where at least one of the following conditions is satisfied:

(i) the chain is infinite;

(ii) there is no interaction, $U=0$;

(iii) $\alpha \rightarrow \infty$, corresponding to interactions between point charges.

None of these criteria is fulfilled in realistic cases of interest. With the exception of carbon nanotubes, experimental molecular junctions involve relatively short $(<10 \mathrm{~nm})$ molecular chains. ${ }^{2}$ The Coulomb interaction is of the same order of magnitude as the kinetic terms ${ }^{21,22}$ and certainly not negligible. Even then, the electron density would still be uniform if the electron-orbitals are reduced to points $(\alpha \rightarrow \infty)$ regardless of the presence of image charges. Therefore, one may say that the nonuniformity of the density comes from the lateral extension of the electronic clouds, the $p$ - or $s$ states. This shows, once again, the need for a threedimensional treatment of the electronic structure.

The exact shape of the electron density is determined by (i) the strength of the Coulomb interaction, $U / t$; (ii) the spread of the electronic orbitals, $\alpha$; and (iii) the geometry of the system, expressed in our model by the length $(n-1) a$ of the molecule and by the distance $d$ between the electrodes and the molecule.

All these factors contribute to yield, instead of the uniform density condition (11), the relation

$$
\epsilon_{n}=-\frac{1}{2} \sum_{m=1}^{N} U_{n, m}\left(1-\frac{\delta_{n, m}}{2}\right)+f_{L}(n),
$$

where $f_{L}(n)$ is a function of the position in the chain; its dependence on $n$ is responsible for the nonhomogeneity of the electronic density. It is of interest to understand the effects of each of these parameters separately.

Coulomb interaction. Starting from the noninteracting case where the electronic density is uniform, increasing $U$ results in an increase of the Friedel oscillations until the electrons start to localize. In the limit $U \rightarrow \infty$, half filling leads to a Mott insulator and one recovers a constant electron density.

Spread of the electronic orbitals. When the orbitals are reduced to points (point charge limit), $\alpha \rightarrow \infty$, the electronic density is uniform. Indeed, in this particular limit the electron-ion and electron-electron interactions have the same form, restoring the electron-hole symmetry of the molecular Hamiltonian. Accounting for a spread of the orbitals increases slowly the amplitude of the Friedel oscillations.

Geometry of the molecular junction. Two effects need to be distinguished: (i) the finite size effects and (ii) the imagecharge effects. In Fig. 2, the dashed curve shows the density for the very same system used to obtain the other two curves, except that now no metallic electrodes are present. Therefore, the dashed curve contains only the finite size effects. From this particular example, one sees that both effects contribute to the Friedel oscillations and that, in order to get the correct density, image interactions should be included unless the electrode-molecule distances are large. In fact, in the example of Fig. 2, the contribution to the oscillations due to the image charges is the larger one. The Coulomb interaction with the electrodes described by the image charges is therefore crucial to estimate transport properties of molecular wires or metallic constrictions. We have observed, as expected, that finite size effects alone tend to disappear when the chain size is increased. In contrast, the effect of image charges tends to become more important: the amplitude of the Friedel oscillations increases with the system size due to the presence of the two metallic electrodes. This tendency should continue until, for long wires, which we do not consider here, the oscillations occur predominantly near the edges.

Finally, the dotted curve of Fig. 2 shows the electron density with metallic electrodes calculated within the Hartree-Fock approximation; it is in very good agreement with the exact result. We have performed calculations for different values of $U$ up to $U=4 t$ and observed that, as far as the electron density is concerned, the Hartree-Fock approximation gives reasonable results. This allow us to use this mean-field approach for the study of the electrostatic profile in biased molecular wires (Sec. IV) and their transport properties (Sec. V). 


\section{ELECTROSTATIC POTENTIAL IN BIASED MOLECULAR WIRES}

The application of the screened on-site electrostatic potential to calculations of conductance properties is useful only within a single electron theory, e.g., in the mean-field description of the process. This is how this concept was applied in the calculation of Mujica et al. ${ }^{16}$ and in the density functional theory. ${ }^{17,18}$ Here, our calculations are done using the Hartree-Fock approximation applied to the molecular Hamiltonian (9). In the previous section, by comparing the mean-field electron density with the exact one, we have already shown that the approximate result is reasonable in the range of parameters proper to conjugated molecules. More precisely, the Hartree-Fock density is in very good agreement with the exact results for values of $U$ up to, approximately, $2 t$ (cf. Fig. 2) and remains of reasonable accuracy up to values of about $4 t$. In the following we present only results for $U=t$, but the same qualitative picture arises also for larger values of $U$.

Starting from the initial tight-binding model (9), we build an effective Hamiltonian by solving self-consistently the usual Hartree-Fock equations ${ }^{21}$ leading to

$$
\begin{aligned}
H_{\mathrm{eff}}= & \sum_{n, \sigma} \epsilon_{n}(V) c_{n, \sigma}^{\dagger} c_{n, \sigma} \\
& +\sum_{n, n^{\prime}, \sigma} t_{n, n^{\prime}}(V)\left(c_{n^{\prime}, \sigma}^{\dagger} c_{n, \sigma}+\text { h.c. }\right),
\end{aligned}
$$

where $V=\mu_{l}-\mu_{r}$. The chemical potentials will always be chosen such that $\mu_{l}=-\mu_{r} . \epsilon_{n}(V)$ is the effective on-site potential which includes the ionic attraction and the electron-electron repulsion incorporated within a meanfield picture as well as the on-site potential (10). $t_{n, n^{\prime}}(V)$ is the effective hopping matrix element which includes the exchange terms. Note that a vanishing of exchange integrals in the ZDO approximation is not equivalent to a Hartree approximation. Indeed, a mean-field approximation of (9) still contains exchange terms of the form $-\frac{1}{2} \Sigma_{n \neq n^{\prime}} U_{n, n^{\prime}}\left\langle c_{n, \sigma}^{\dagger} c_{n^{\prime}, \sigma}\right\rangle c_{n^{\prime}, \sigma}^{\dagger} c_{n, \sigma}$ which, due to the longrange part of the Coulomb potential (4), include long-range hopping.

Without Coulomb interactions, the electrostatic potential is given by the ramp defined in (10), i.e., by the potential in the absence of a molecule. This linear profile has been used sometimes in the literature to study nonlinear current-voltage characteristics. $^{27,28}$

In the presence of Coulomb interaction, the linear profile is changed and the screened electrostatic potential, $E_{n}(V)$, is given by the difference between the on-site term with and without bias voltage

$$
E_{n}(V)=\epsilon_{n}(V)-\epsilon_{n}(0) .
$$

A typical example is shown in Fig. 3 for a chain with 20 sites. We see two main features: (i) screening is not very efficient and, consequently, there is only a small potential drop at the interfaces but a finite slope of the potential along the entire molecule; (ii) there are substantial Friedel oscillations along the profile.

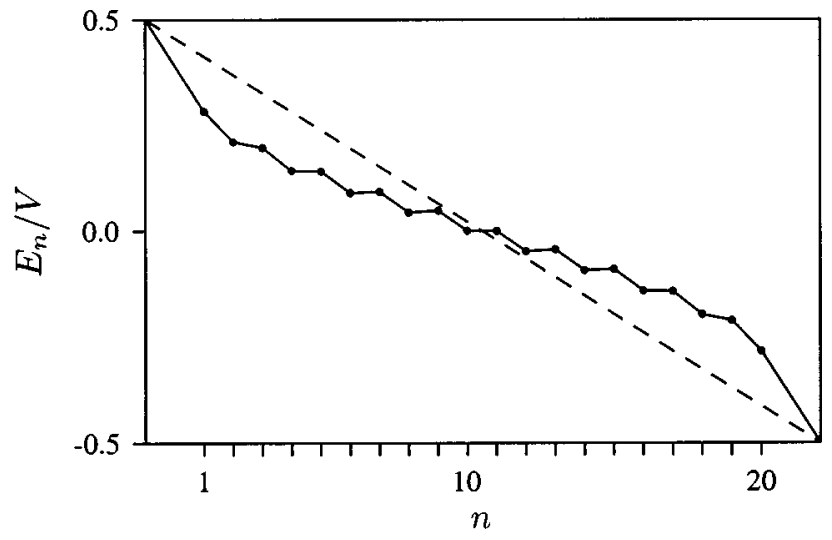

FIG. 3. Electrostatic potential profile for a carbon wire $[s=1$ in Eq. (5)] with $N=20$ sites. The parameters are $\alpha=4.5 / a^{2}, U=t, V=\mu_{l}-\mu_{r}=t$, and $d=2 a$. The dashed curve is the unscreened potential without molecule (ramp potential). The full curve is the screened potential in the presence of the molecular wire. It shows a small decrease in slope along the entire molecule with substantial Friedel oscillations.

Our results differ from those of Mujica et al. ${ }^{16}$ despite the fact that the two models are closely related. These differences mostly come from the fact that we solve the Poisson equation without resorting to a one-dimensional approximation. As recently shown by us within a classical model, ${ }^{19}$ a three-dimensional treatment of the electrostatics is necessary, and in fact leads to the identification of the lateral thickness of the molecule as a new generic attribute that determines the potential profile. In Fig. 4, we show a comparison between our results and the calculations of Damle et al. ${ }^{18}$ for a chain of six gold atoms. It is important to stress that we did not try to fit the $a b$ initio curve but, instead, we simply chose a reasonable set of parameters. Note, however, that we include the response of infinite leads while the $a b$ initio calculations take only small metal clusters into account. Furthermore, the asymmetry in the latter case indicates that the ab initio calculations result in a charged molecule, while in our case the molecular wire always remains neutral. Other ab initio calculations ${ }^{17,29,30}$ on similar models are also in agreement with our observations.

To conclude this section, we summarize our main results: in the relevant range of parameters, screening is not

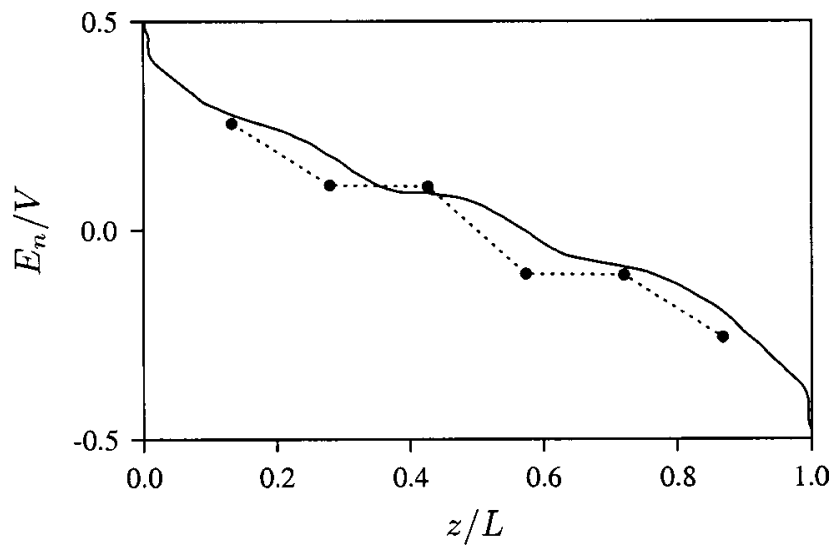

FIG. 4. The electrostatic potential profile for a gold wire $[s=0$ in Eq. (5)] with $N=6$ sites obtained from Hartree-Fock calculations is shown by the filled circles. The parameters used are $d=0.9 a, \alpha=4.5 / a^{2}$, and $U=t$. For comparison the full curve shows the ab initio results taken from Ref. 18. 
very efficient in the wire and the drop of the potential occurs along the entire molecule. Additionally, substantial Friedel oscillations are present in the electrostatic profile. Our results are in good agreement with recent ab initio results. ${ }^{17,18}$ When compared with the results of Ref. 16, our treatment stresses the need to study the full three-dimensional problem, as far as the electrostatics of the molecular junction is concerned.

In the next section, we discuss consequences of the screening effects for the current-voltage characteristics of molecular wires studied within the Landauer formalism.

\section{CURRENT-VOLTAGE CHARACTERISTICS OF A MOLECULAR WIRE IN THE WEAK TUNNELING CONTACT LIMIT}

In a model where only the first and the last atom of a molecular chain couple to the corresponding metal leads and at $T=0$, the Landauer conduction formula yields ${ }^{3,31}$

$$
I=\frac{2 e}{\pi \hbar} \int_{\mu_{r}}^{\mu_{l}} d E\left|G_{1 N}(E, V)\right|^{2} \Delta_{l}(E, V) \Delta_{r}(E, V),
$$

where $V=\mu_{l}-\mu_{r}$ and $\Delta_{l / r}$ are the spectral functions for the left and right reservoirs. The molecule-lead coupling only occurs at sites 1 and $N$ and $G_{1 N}$ is the matrix element of the exact Green function of the molecular junction between these sites (cf. Fig. 1).

This equation can be understood as a special case of the Landauer formula ${ }^{32}$ adapted to the case of bad contacts, where it is possible to use second-order perturbation theory in the tunneling matrix element. ${ }^{31}$ In the limit of "extremely" bad contacts of interest here, the Green function of the system in formula (15) may be replaced by the Green function of the isolated molecular wire, $G_{1 N}^{0} .{ }^{33}$ Moreover, assuming that the product of spectral densities does not significantly depend on energy in the range between $\mu_{l}$ and $\mu_{r}$, one gets

$$
I=\frac{2 e}{\pi \hbar} \Delta_{0}^{2} \int_{\mu_{r}}^{\mu_{l}} d E\left|G_{1 N}^{0}(E, V)\right|^{2},
$$

where $\Delta_{0}$ is the spectral density at zero bias.

From this simplified equation, we can calculate the current-voltage characteristics of the molecular junction, using only the Green function of the isolated molecular wire, evaluated in the presence of the electrostatic potential created by the metallic electrodes. Examples are given in Fig. 5 for increasing Coulomb strength $U$ starting from the noninteracting case where the on-site potential is given by the ramp (10).

In all cases, the $I-V$ curves have a staircase structure which is a common feature in the weak tunneling limit ${ }^{18,27,34}$ and simply reflects the discreteness of the molecular electronic spectrum. Indeed, an increase of the bias potential corresponds to an increase of the window of integration in formula (16). Therefore, a jump in the $I-V$ curves means that one more discrete molecular level enters this window of integration.

It is interesting to note that Fig. 5 shows also wide regions of negative differential resistance, in particular in the noninteracting case. Within our simple formulation, they can

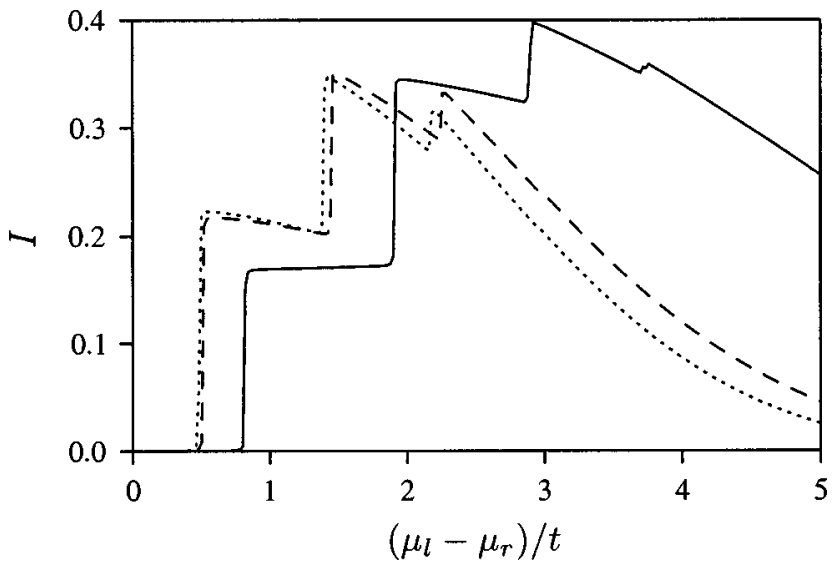

FIG. 5. Current-voltage characteristics for a carbon molecule $(s=1)$ with $N=12, d=2 a, \alpha=4.5 / a^{2}$, and for different Coulomb interaction strengths $U / t=0,0.1,1$ are depicted by the dotted, dashed, and full line, respectively. The current is given in units of $(2 e / \pi \hbar) \Delta_{0}^{2} / t$.

be explained by the localization of charges induced by a strong enough electric field: at sufficiently high bias voltage, charge carriers are localized at one end of the chain, resulting in a decrease of the current. This could be an artifact of our weak molecule-electrode coupling model; however, negative differential resistance has been found in recent experiments, ${ }^{35}$ and electric field-induced localization could be a way to understand these experimental findings.

The Coulomb interaction has two main effects on the $I-V$ characteristics. First, the positions of the currentvoltage steps are shifted to higher voltages reflecting the displacement of the molecular levels to higher energy with increasing Coulomb interaction. Second, the localization of the charges due to a strong electric field is partially compensated by the electron-electron repulsion. These screening effects attenuate the negative differential resistance effects (cf. Fig. 5). These observations are similar to those made by Mujica et al. ${ }^{27}$ where a Hubbard model was studied at the Hartree level.

\section{CONCLUSION}

We have addressed the problem of calculating transport properties of a molecular wire bridging two semi-infinite metallic electrodes. A first important part of this task is to determine the electrostatic potential profile through the biased wire. ${ }^{14-18}$ Indeed, it is of importance to know how screening effects modify the ramp potential [Eq. (10)] existing in the absence of the organic molecule.

This work and our earlier paper ${ }^{19}$ resolve discrepancies between answers available in the literature. On the one hand, a tight-binding model combined with a one-dimensional Poisson equation gives a strong screening version of the problem: the drop of the potential occurs at the interfaces and the potential is therefore almost flat within the molecule. ${ }^{16}$ On the other hand, recent $a b$ initio results give a weak screening version: no substantial drop at the interfaces but rather a decrease along the entire molecule together with substantial Friedel oscillations. ${ }^{17,18}$ 
In this paper, we have proposed a modified tight-binding model to address this question. It is based on three main ingredients. (i) We introduce a three-dimensional Coulomb potential [Eq. (4)] which includes the image interaction with the two metallic electrodes assumed to have planar surfaces. (ii) The electrons localized at atomic sites are modeled by Gaussian-type orbitals of finite lateral extent [Eq. (5)]. (iii) The positive background is assumed to be a set of point charges localized on the atomic sites. With these three ingredients, it is possible to evaluate the various terms of our model Hamiltonian: the on-site energy [Eq. (7)] and the electron-electron interaction [Eq. (8)]. They are all functions of the position on the chain due to the finite size of the system and the image charges induced by the electrodes. All our calculations are done in the weak tunneling limit, assuming bad contacts.

This model yields an electronic density that is nonuniform already in the absence of a bias potential - the electrons prefer to be in the center of the wire-and displays pronounced Friedel oscillations (Fig. 2). These characteristics are due to the fact that our model does not fulfill, in general, the electron-hole symmetry. ${ }^{24-26}$ In the presence of an applied voltage the electrostatic potential profile does not drop appreciably at the interfaces but rather, in accordance with the ab initio results of Refs. 17 and 18, it decreases along the molecule with substantial Friedel oscillations appearing along the entire profile (Figs. 3 and 4). Our results are different from the ones of Ref. 16, where a one-dimensional Poisson equation was used. This disagreement stresses the need to perform a three-dimensional calculation, as done here, to properly describe the electrostatic properties of the molecular junction.

Finally, the current-voltage characteristics are obtained within the same Hartree-Fock calculation using the Landauer formula (i.e., neglecting electronic correlation effects and assuming coherent tunneling). It shows a staircase structure (Fig. 5), as is common in the weak tunneling limit. ${ }^{18,27}$ Zones of negative differential resistance are found due to charge localization induced by the electric field. The main effects of the Coulomb interaction, within our approximations, are, on the one hand, a shift to higher energies of the position of the current-voltage steps and, on the other hand, a partial compensation of the localization of the electrons diminishing the negative differential resistance effects in agreement with a previous study. ${ }^{27}$

In closing, it is important to stress some limitations of our model. On the one hand, we consider coherent transport, assuming that the electrons are transferred from one lead to the other in a single quantum-mechanical process. This is a good approximation if the tunneling time is much less than the inelastic scattering time. For organic molecules, this transit time could be of the same order of magnitude as the intramolecular vibronic relaxation time, especially in the weak contact limit employed here. ${ }^{3}$ In this case, part of the current could be due to sequential tunneling, where the molecular wire would be successively charged and discharged. This important issue remains to be studied further. We have neglected charging effects assuming the molecule to remain neutral. At high voltage, this approximation could fail. ${ }^{27}$ The average charge number of the molecule could increase in analogy with Coulomb blockade phenomena observed in mesoscopic metallic double-tunnel junctions and quantum dots, ${ }^{36}$ and, more recently, in multiwall carbon nanotubes. ${ }^{37}$

A proper handling of the full problem requires treating the wire as an open system dynamically coupled to the electrodes and to the vibronic degrees of freedom. This program is far beyond the scope of the present work.

\section{ACKNOWLEDGMENTS}

The authors have benefited from useful discussions with P. Hänggi, S. Kohler, J. Lehmann, and S. Yaliraki. Three of us (H.G., G.L.I., and A.N.) would like to thank the Institute for Theoretical Physics at UCSB for hospitality during the workshop on "Nanoscience" where this work was started. This research was supported in part by the National Science Foundation under Grant No. PHY99-07949, by the Volkswagen-Stiftung under Grant No. I/77217, by the Israel Science Foundation and by the Israel Ministry of Science.

${ }^{1}$ A. Aviram and M. A. Ratner, Chem. Phys. Lett. 29, 277 (1974)

${ }^{2}$ C. Joachim, J. K. Gimzewski, and A. Aviram, Nature (London) 408, 541 (2000).

${ }^{3}$ A. Nitzan, Annu. Rev. Phys. Chem. 52, 681 (2001).

${ }^{4}$ Special issue on Processes in Molecular Wires, edited by P. Hänggi, M. Ratner, and S. Yaliraki, Chem. Phys. 281, 111-487 (2002).

${ }^{5}$ M. A. Reed, C. Zhou, C. J. Muller, T. P. Burgin, and J. M. Tour, Science 278, 252 (1997).

${ }^{6}$ C. Kergueris, J.-P. Bourgoin, S. Palacin, D. Esteve, C. Urbina, M. Magoga, and C. Joachim, Phys. Rev. B 59, 12505 (1999).

${ }^{7}$ X. D. Cui, A. Primak, X. Zarate, J. Tomfohr, O. F. Sankey, A. L. Moore, T. A. Moore, D. Gust, G. Harris, and S. M. Lindsay, Science 294, 571 (2001).

${ }^{8}$ J. Reichert, R. Ochs, D. Beckmann, H. B. Weber, M. Mayor, and H. v. Löhneysen, Phys. Rev. Lett. 88, 176804 (2002).

${ }^{9}$ E. G. Petrov and P. Hänggi, Phys. Rev. Lett. 86, 2862 (2001); E. G. Petrov, V. May, and P. Hänggi, Chem. Phys. 281, 211 (2002).

${ }^{10}$ J. Lehmann, G.-L. Ingold, and P. Hänggi, Chem. Phys. 281, 199 (2002).

${ }^{11}$ D. Segal and A. Nitzan, Chem. Phys. 281, 235 (2002).

${ }^{12}$ H. M. Pastawski, L. E. F. Foa Torres, and E. Medina, Chem. Phys. 281, 257 (2002).

${ }^{13}$ H. Ness and A. J. Fisher, Chem. Phys. 281, 279 (2002).

${ }^{14}$ S. Datta, W. Tian, S. Hong, R. Reifenberger, J. I. Henderson, and C. P. Kubiak, Phys. Rev. Lett. 79, 2530 (1997).

${ }^{15}$ W. Tian, S. Datta, S. Hong, R. Reifenberger, J. I. Henderson, and C. P. Kubiak, J. Chem. Phys. 109, 2874 (1998).

${ }^{16}$ V. Mujica, A. E. Roitberg, and M. A. Ratner, J. Chem. Phys. 112, 6834 (2000).

${ }^{17}$ N. D. Lang and P. Avouris, Phys. Rev. Lett. 84, 358 (2000).

${ }^{18}$ P. S. Damle, A. W. Ghosh, and S. Datta, Phys. Rev. B 64, 201403 (2001).

${ }^{19}$ A. Nitzan, M. Galperin, G.-L. Ingold, and H. Grabert, J. Chem. Phys. 117, 10837 (2002).

${ }^{20}$ A. W. Ghosh, private communication (2002).

${ }^{21}$ L. Salem, Molecular Orbital Theory of Conjugated Systems (Benjamin, London, 1966).

${ }^{22}$ D. Baeriswyl, D. K. Campbell, and S. Mazumdar, in Conjugated Conducting Polymers, edited by H. Kiess (Springer, Heidelberg, 1992), pp. 7-133.

${ }^{23}$ P. Fulde, Electron Correlations in Molecules and Solids (Springer, Berlin, Heidelberg, New York, 1995).

${ }^{24}$ C. A. Coulson and G. S. Rushbrooke, Proc. Cambridge Philos. Soc. 36, 193 (1940).

${ }^{25}$ A. D. MacLachlan, Mol. Phys. 2, 271 (1959); 4, 49 (1961).

${ }^{26}$ E. H. Lieb, M. Loss, and R. J. McCann, J. Math. Phys. 34, 891 (1993).

${ }^{27}$ V. Mujica, M. Kemp, A. Roitberg, and M. Ratner, J. Chem. Phys. 104, 7296 (1996)

${ }^{28}$ S. Dallakyan and S. Mazumdar, arXiv:cond-mat/0209143. 
${ }^{29}$ H. B. Weber, J. Reichert, F. Weigend, R. Ochs, D. Beckmann, M. Mayor, R. Ahlrichs, and H. v. Löhneysen, Chem. Phys. 281, 113 (2002).

${ }^{30}$ P. Damle, A. W. Ghosh, and S. Datta, Chem. Phys. 281, 171 (2002).

${ }^{31}$ J. Bardeen, Phys. Rev. Lett. 6, 57 (1961).

${ }^{32}$ R. Landauer, IBM J. Res. Dev. 1, 223 (1957); S. Datta, Electronic Transport in Mesoscopic Systems (Cambridge University Press, Cambridge, England, 1995).

${ }^{33}$ A. Onipko, Y. Klymenko, L. Malysheva, and S. Stafström, Solid State Commun. 108, 555 (1998).
${ }^{34} \mathrm{~A}$ finite molecule-lead coupling matrix element would smoothen the sharp steps in Fig. 5.

${ }^{35}$ J. Chen, M. A. Reed, A. M. Rawlett, and J. M. Tour, Science 286, 1550 (1999).

${ }^{36}$ Single Charge Tunneling. Coulomb Blockade Phenomena in Nanostructures, edited by H. Grabert and M. H. Devoret (Plenum, New York, 1992).

${ }^{37}$ M. R. Buitelaar, A. Bachtold, T. Nussbaumer, M. Iqbal, and C. Schönenberger, Phys. Rev. Lett. 88, 156801 (2002). 